\title{
Chryseobacterium indologenes as a Rare Pathogen of Bacteremia in Febrile Neutropenia
}

\author{
${ }^{1}$ Mersin University Faculty of Medicine, Department of Pediatric \\ Oncology, Mersin, Turkey \\ 2 Mersin University Faculty of Medicine, Department of Pediatrics, \\ Mersin, Turkey \\ Cite as: Demir Gündoğan B, Sağcan F, Çağlar Çıtak E. \\ Chryseobacterium indologenes as a rare pathogen of bacteremia \\ in febrile neutropenia. Trends in Pediatrics 2021;2(1):43-6.
}

Begümhan Demir Gündoğan ${ }^{1 \oplus}$, Fatih Sağcan ${ }^{2 \oplus}$, Elvan Çağlar Çıtak ${ }^{1 \oplus}$

Received: 16 October 2020

Accepted: 25 December 2020

Publication date: 30 March 2021

Keywords: Neutropenia, fever, chryseobacterium, bacteremia

\section{Begümhan Demir Gündoğan}

Mersin University Faculty of Medicine, Department of Pediatric

Oncology, Mersin, Turkey

ORCID: 0000-0002-6234-0874

begum-han@windowslive.com

F. Sağcan 0000-0002-4788-3256

E. Ç. Çıtak 0000-0003-1451-1373

\section{ABSTRACT}

Chryseobacterium indologenes (C. indologenes) is nonmotile, oxidase-, and indole-positive gram-negative aerobic bacillus. Immunosuppression, comorbidities, use of broad-spectrum antibiotics are known risk factors for $C$. indologenes-related infections. We report a neutropenic fever caused by $C$. indologenes in a 16-month-old boy who was treated due to the neuroblastoma. According to the antimicrobial susceptibility test result, he was treated with cephaperazone/sulbactam.

\section{INTRODUCTION}

Chryseobacterium indologenes (C. indologenes) is aerobic, non-fermentative, non-motile, oxidase- and indole- positive, Gram-negative bacillus. ${ }^{1} \mathrm{C}$. indologenes is known to cause different types of infections such as bacteremia, pneumonia, meningitis and shunt infection, especially in patients hospitalized longterm with indwelling devices and using long-term broad-spectrum antibiotics. C. indologens can also be the cause of serious infections in immunocompromised patients. With increased use of colistin and tigecycline against carbapenem- resistant microorganisms such as Acinetobacter baumannii, Escherichia coli and Klebsiella pneumoniae, there is an increase in the incidence of infections associated with
Chryseobacterium spp..$^{1,2}$ Herein, we describe a neuroblastoma patient with febrile neutropenia who had a bloodstream infection due to $C$. indologenes which was successfully treated with cephaperazone/ sulbactam..$^{1,2}$

\section{CASE REPORT}

A 16-month-old boy was admitted to our hospital with complaints of high fever, weakness and pallor. On physical examination, a $2 \mathrm{~cm}$ palpable mass on the right upper quadrant was detected. An adrenal mass was detected on the right side of abdomen on abdominal computed tomography. The patient was diagnosed with neuroblastoma and the neuroblastoma protocol of Turkish Pediatric Oncology Group, 
including A9 (vincristine $1,5 \mathrm{mg} / \mathrm{m}^{2}$ on days 1 and 5; dacarbasine $200 \mathrm{mg} / \mathrm{m}^{2}$ on days 1-5; Ifosfamid 1500 $\mathrm{mg} / \mathrm{m}^{2}$ on days $1-5$; adriamycin $30 \mathrm{mg} / \mathrm{m}^{2} 65$ on days 4 and 5 ) and A11 (cyclophosphamide $300 \mathrm{mg} / \mathrm{m}^{2}$ on days $1-5$, etoposide $80 \mathrm{mg} / \mathrm{m}^{2}$ on days $1-4$; cisplatin $30 \mathrm{mg} / \mathrm{m}^{2}$ on days $1-5$ ) was started alternately for each 21 days.

Febrile neutropenia was diagnosed after the fourth course of treatment. The patient underwent a detailed physical examination and blood culture was obtained before starting antibiotic and cephaperazone/sulbactam monotherapy was begun. Vancomycin was added to the treatment after the fever persisted for 72 hours. On the fifth day of fever, C. indoligenes was isolated using the BACTEC 9120 system (Becton-Dickinson Diagnostic Systems, USA). Identification and antimicrobial susceptibility testing of the isolate was performed using the Vitek ${ }^{\circledR}$ system (bio-Mérieux, France) according the recommendation of the Clinical and Laboratory Standards Institute. ${ }^{3}$ According to the antibiotic susceptibility test result, treatment regimen was not changed as C. indologens were sensitive to cephaperazone/sulbactam. The patient became afebrile on the seventh day of cephaperazone/sulbactam treatment and treatment was completed before any complications developed.

\section{DISCUSSION}

Children treated with solid tumors or hematological malignancies have an increased risk of infections. In these patients, the incidence of bacteremia is still reported as $10-40 \%$, and mortality rates reach to 9-24\% due to serious complications. ${ }^{4,5}$

With the developing modern culture systems, previously unidentifiable microorganisms have become identifiable and in many infectious diseases, new microorganisms have been reported as causative agents. One of them is $C$. indologenes, a member of the Chryseocabterium spp. The SENTRY Antimicrobial Surveillance Program is a worldwide study that monitors the susceptibility and resistance of bacteria and fungi to antibiotics based on results from more than 119 sentry hospitals and laboratories in North America, Latin America, Europe and the Asia-Pacific region. According to the data of this study, which was carried out between 1997 and 2001, Chryseobacterium spp. appear to be a rare pathogen representing only $0.27 \%$ of non-fermentative Gramnegative bacilli and $0.03 \%$ of all bacterial isolates collected from adults and children. In addition, in this study, it was reported that Chryseobacterium spp. can only be isolated in $0.10 \%$ of the culture samples taken from the respiratory tract and $0.03 \%$ of the blood culture samples. ${ }^{2,6}$

Bacteremia due to $C$. indologenes is becoming increasingly common. ${ }^{7}$ It has been reported that bacteremias due to $C$. indologenes are associated with nosocomial pneumonias, biliary tract infections, peritonitis, urinary tract infections, surgical wound infections, cellulitis, intravascular catheterrelated bacteremia and primary bacteremia. ${ }^{2,8} \mathrm{Also}$ it has been reported that most cases with $C$. indologenes sepsis, have either severe underlying diseases such as malignancy or diabetes mellitus, either taking long-term broad-spectrum antibiotic therapy or using indwelling devices. ${ }^{9,10}$

C. indologenes has been rarely reported to be a causative agent in febrile neutropenia in children. In their studies evaluating Flavobacteriaceae bacteria in children, Cooper et al found that nine out of the $13 \mathrm{fla}$ vobacteiaceae growths in the last 20 years were $C$. indologenes ${ }^{(11)}$. Five of nine cases with growth of C. indologenes in their culture media were immunosuppressive patients (two patients with acute lymphoblastic leukemia and one patient each with hemophagocytic lymphohistiocytosis, aplastic anemia and acute myeloid leukemia) in that study. Although it was reported that three cases had neutropenic fever, it was thought that leukostasis in T-ALL patient with fever at the stage of diagnosis may be due to the presence of lymphoblasts in peripheral blood, although it was not mentioned in the article, a total of four cases had neutropenic fever. Only one of these five patients was not catheterized and diagnosed with febrile neutropenia. To our knowledge, C. indologenes were not identified as the causative agent of febrile neutropenia, except in the cases mentioned in this study. This shows us that C. indologenes are rarely seen among microorganisms that cause bacteremia in febrile neutropenia. In our case, $C$. indologenes was isolated from blood cultures, and our case had not central catheter, 
which was among the risk factors for $C$. indologenes bacteremia.

An empirically effective drug selection in infections caused by $C$. indologenes is difficult due to the limited antimicrobial susceptibility of the organism. Studies have shown that C.indologenes is susceptible to piperacillin-tazobactam, piperacillin, cefoperazone, ceftazidime, cefepime, cefpiroma, minocycline, rifampicin, TMP-SMZ and new fluoroquinolones (garenoxacin, gatifloxacin, levofloxacin); conversely, resistant to extended- spectrum penicillins, first and second generation cephalosporins, carbapenems, ceftriaxone, aztreonam, ticarcillin clavulonate, chloramphenicol, erythromycin, clindamycin, aminoglycosides, tetracycline and teicoplanin. $C$. indologens can produce a variety of $\beta$-lactamase species, which contribute to multiple antibiotic resistances. ${ }^{12}$

We started the treatment with cefaperazone/sulbactam, which is an antipseudomonal antibiotic as empirically recommended in febrile neutropenia guidelines. ${ }^{13}$ As our patient did not have a blood culture result at 72 hours and his fever continued, vancomycin, an antibiotic in the glycopeptide group, was added to the treatment as stated in the guidelines. On the 5th day of the treatment, the treatment was continued without antibiotic change due to bacterial sensitivity to cefaperazone/sulbactam according to the blood culture and antibiogram results and the treatment was successfully completed without any complications.

In our case, cephaperazone/sulbactam treatment, which was started empirically, was continued after the antibiogram showed its antimicrobial sensitivity and the treatment was successfully completed without any complication. We think that blood culture results should be monitored closely and treatment options should be adjusted according to antibiotic susceptibility in these patients.

In conclusion, this case shows us that although the majority of $C$. indologenes infections are associated with indwelling catheter use, non-catheter-related bacteremia can also be a causative factor, and C. indologenes should be considered as a possible cause of febrile neutropenia in children.
Conflict of Interest: The authors declared no potential conflicts of interest with respect to the research, authorship, and/or publication of this article.

Funding: The authors received no financial support for the research, authorship, and/or publication of this article.

Informed Consent: Parents of the patient provided informed consent to publish the report.

\section{REFERENCES}

1. Nemli SA, Demirdal T, Ural S. A case of healthcare associated pneumonia caused by Chryseobacterium indologenes in an immunocompetent patient. Case Rep Infect Dis. 2015;2015:483923 https://doi.org/10.1155/2015/483923

2. Celkan T, Ozkan A, Apak H, Diren S, Can G, Yuksel L et al. Bacteremia in childhood cancer. J Trop Pediatr. 2002;48:373-7.

https://doi.org/10.1093/tropej/48.6.373

3. Clinical and Laboratory Standards Institute (2014) Performance standards for antimicrobial susceptibility testing. Twenty-Fourth informational supplement. M100-S24. CLSI, Wayne, PA.

4. Chen FL, Wang GC, Teng SO, Ou TY, Yu FL, Lee WS. Clinical and epidemiological features of Chryseobacterium indologenes infections: analysis of 215 cases. J Microbiol Immunol and Infect. 2013;46: 425-32. https://doi.org/10.1016/j.jmii.2012.08.007

5. Aslan S, Citak EC, Yis R, Degirmenci S, Arman D. Bacterial spectrum and antimicrobial susceptibility pattern of bloodstream infections in children with febrile neutropenia: experience of single center in southeast of Turkey. Indian J Microbiol. 2012;52:203208

https://doi.org/10.1007/s12088-011-0210-6

6. Kirby JT, Sader HS, Walsh TR, Jones RN. Antimicrobial Susceptibility and Epidemiology of a Worldwide Collection of Chryseobacterium spp: report from the SENTRY Antimicrobial Surveillance Program (19972001). J Clin Microbiol. 2004;42:445-8. https://doi.org/10.1128/JCM.42.1.445-448.2004

7. Hsueh PR, Teng LJ, Yang PC, Ho SW, Hsieh WC, Luh KT. Increasing incidence of nosocomial Chryseobacterium indologenes infections in Taiwan. Eur J Clin Microbiol Infect Dis. 1997;16:568-74. https://doi.org/10.1007/BF02447918

8. Corbella M, Brandolini M, Cambieri P, et al. A catheterrelated bloodstream infection caused by Chryseobacterium indologenes successfully treated with antibiotic-lock rescue therapy. New Microbiol. 2017 Jul;40(3):223-5.

9. Deng L, Li MF, Li YH, Yang JL, Zhou X. Chryseobacterium indologenes in four patients with leukemia. Transpl Infect Dis. 2015;17:583-7. https://doi.org/10.1111/tid.12400

10. Esposito S, Russo E, De Simone G, Gioia R, Noviello S, Vitolo $\mathrm{M}$ et al. Transient bacteraemia due to Chryseobacteriumindologenesinan immunocompetent patient: a case report and literature review. J Chemother 
2014;27:324-9.

https://doi.org/10.1179/1973947814Y.0000000206

11. Cooper S, Levy I, Ben-Zvi H, Ashkenazi-Hoffnung L, BenShimol S, Shachor-Meyouhas Y et al. Flavobacteriaceae Bacteremia in Children: A Multicenter Study. Pediatr Infect Dis J 2019;38:1096-9.

https://doi.org/10.1097/INF.0000000000002449

12. Lin YT, Jeng YY, Lin ML, Yu KW, Wang FD, Liu CY. Clinical and microbiological characteristics of Chryseobacterium indologenes bacteremia. Journal of Microbiology,
Immunology and Infection, 2010;43(6):498-505. https://doi.org/10.1016/S1684-1182(10)60077-1

13. Lehrnbecher $T$, Robinson $P$, Fisher $B$, Alexander $S$, Ammann RA, Beauchemin $M$ et al. Guideline for the Management of Fever and Neutropenia in Children With Cancer and Hematopoietic Stem-Cell Transplantation Recipients: 2017 Update. J Clin Oncol. 2017;35:2082-94.

https://doi.org/10.1200/JCO.2016.71.7017 